\title{
Split and Schedule (SS): A Progressive Lifetime for Barrier Coverage using Relay Clusters in Wireless Sensor Network
}

\author{
C. N. Abhilash \\ Department of CSE, UVCE \\ Bangalore University, \\ Bengaluru, Karnataka, India
}

\author{
S. H. Manjula \\ Department of CSE, UVCE \\ Bangalore University, \\ Bengaluru, Karnataka, India
}

\author{
K. R. Venugopal \\ Department of CSE, UVCE \\ Bangalore University, \\ Bengaluru, Karnataka, India
}

\begin{abstract}
In wireless sensor networks, barrier coverage is one of the major challenge for high density area. To overcome this challenge, coverage control is the key solution for making more efficient and can program the sensors in active or idle state to maintain network coverage. Also, the random deployment with clustering of detection nodes plays a crucial role in the presence of an obstruction else that can become a viable route for attackers. In this paper, two strategies are proposed to resolve the problem of barrier coverage and to improve lifespan of the network. A Split and Schedule (SS) algorithm is proposed and the formation of clusters overcomes limitations with better solution. By comparing with other efficient algorithms, proposed SS algorithm sustains the better quality of network coverage and enhances the longevity of the sensor network.
\end{abstract}

\section{General Terms}

Wireless Sensor Networks, Coverage Problem

\section{Keywords}

Barrier Coverage, Cluster, Delay, Relay node, Network Lifetime, Sensor network

\section{INTRODUCTION}

Wireless sensor network (WSN) has become the supreme technologies in recent trend deployed in different application areas. They are proving to be an element of everyday life. Modern WSNs [1] tend to get an ever more diverse set of nodes that link within the same grid with different design and potential. Recent advances in miniaturization of hardware, communication technology and low cost networks with hundreds of small battery-powered sensors, wirelessly connected, have led to a wide range of innovative applications. Applications are border surveillance, intelligent internal security and environmental monitoring [2]. The presence of WSN Mobile has led to a very challenging task to detect information.

A mobile relay node (MRN) has the ability to communicate shortly and collect data through the sensor nodes while moving along the path. Using MRN increases the useful life of the network as it will consume more energy when distributing the task assigned to a specific sensor. The nodes nearest to the receiver must transmit the data from the nodes of the sensors that are beyond the range. This leads to an overload check of nodes during MRN communication to the sink. Replacing the MRN can also be performed at regular intervals of time by observing the MRN energy levels at the base station. This charging energy cannot be carried out in the nodes of the sensors that are fixed in predetermined positions.
In this paper, the issues of barrier coverage and connectivity in the network are discussed despite obstruction across the traversed path. Solutions are proposed using a split and schedule algorithm (SS) and few heuristics to achieve better network lifespan with less consumption of power using MRN in WSN. First, all nodes are subdivided into a number of clusters related to their rate of data generation and position [13]. Formerly, around a distinct cluster, planning is performed when a node visit is to be performed for the MRN, reducing the overload of travel back and forth to reach long and distant nodes. Finally, solutions to plan cluster node visits are added and sent to the path of the mobile forwarding node so that each node can be reached at tolerable frequencies to avoid overflow of storage. The metrics discussed are analyzed with the rate of packet delivered without loss to a given MRN speed and the required speed to completely overflow the storage. The implementation of the proposed SS algorithm achieves high performance and even in case of obstruction there is an improvement in the longevity of the network.

The next sections of the paper are organized as follows: The Section 2 presents the related work, followed by description of the barrier coverage and MES problem in Section 3 and the Constraints for MRN in Section 4. The proposed SS algorithm is explained in Section 5. Further Simulation setup and results are defined in Section 6. Lastly, the conclusion is described in Section 7.

\section{RELATED WORK}

All Conventional WSN prototypes depend on the idea that the system is large, so that two nodes of sensors can share information across multiple session modules. Consequently, most times it is believed that sensors are static and that portability is non-relevant option. In recent times, it recalls the developments in research in [1] Ad hoc networks also called MANET and delay tolerance networks (DTNs) [2], mobility has also been launched to wireless sensor networks [3] [4]. The motion design inherited from WSN is linked, i.e., messages are generated by nodes and the recipient gathers all the messages. Consequently, the sensors closest to the receiver are richer in energy than others, and the approach to initial energy consumption is called channeling effect [5], the neighboring receiver nodes represent the traffic jam. MRN helps to reduce the effect of channeling because it visits different areas of the network and extends the use of energy evenly, also in the state of a dense architecture of WSN [6] [7]. A system with nodes displaced was presented for topology control management in [8]. Specific, pre-define, intellectual, less-weight, and unique traffic (PILOTs) 
managing sensor nodes are considered to restore connectivity of network in case of unreliable connections. The PILOT node travels to areas where the node association is not stable or fails and works as a link. As a result, they effectively alter the WSN topology to improve the exchange of information reliability and energy efficiency. Calculations for the location of the nodes moved to improve system availability have been explored in [9] [10]. Nodes displaced can be used to resolve the coverage area problem. For this situation [11] [12], the essential point does not guarantee the availability of the system, but maintain a strategic distance from the split cover zone where the thickness of the sensor nodes is insufficient to portray or adequately recognize an accident.

The MES [14] problem in the sensor network is considered NP-complete and calculations are demonstrated using the three heuristics. First, in the calculation of the first time limit (EDF), the sensor node is first reached with the nearest limit. To improve the EDF, in the next calculation, EDF is used with prediction mechanism, instead of reaching a sensor node with an initial limit; this calculation considers the set $k$ probability of nodes with minimum limits and selects the node that requires more timely completion time. The third calculation is the calculation of the sum of the sums of the minimum weight (MWVS) that considers the deadlines with the weights and also the distance between the nodes to determine the programming of the achievement of the nodes. The MWVS calculation achieves the best result compared to the last proposed calculations. But "back and forth" changes often occur with large nodes.

The network of sensors can also be improved through mobility nodes. Xing et al. [15] studied the target detection for mobility support in sensor network. They have developed reactive mobility to improve the performance of the detection target in WSN. Mobile and static sensors work together and move responsive to vital performance. Wang et al. [16] found coverage holes using Voronoi diagrams depends on the theory of movement of sensor nodes from the dense zone to the slightly deployed region. They also presented a mobility support system for field coverage that can be integrated and improved with fewer mobile nodes. An optimal algorithm was used to estimate coverage contributions by exploring the capacity of mobile nodes with longer network life.

\subsection{Objectives of the System Proposed}

The Split and Schedule (SS) algorithm is proposed to resolve the drawback in Mobile Element Scheduling (MES) [13] that focuses on achieving the MRN on individual node to prevent loss of data caused by overflow in sensor storage. By using the SS algorithm, before dividing every node in network into multiple clusters, and every node in the same cluster has the same union term to reduce power consumption. So, to find a solution for the scheduling problem of an MRN inside a single cluster, the traveling salesman problem (TSP) is a good option, where a less costly path should reach each node exactly once to improve network connectivity. Lastly, visiting schedules for individual cluster are combined to generate the full list. Most researchers have suggested that the movement as a better purpose to the difficulty of sensing data. As the nodes moving in the network can hold and send the data implemented to the nodes in network. To get an overview of WSN forwarding nodes and coverage features, let's first consider the network model with a barrier coverage problem in the WSN as shown in Fig. 1. The attacker's (red arrows) can enter the network, where the intersection of the nodes is not completely covered, so a network with less node density can be advantageous with the MRNs because they are very useful and also play an important solution in an isolated node network of sensors. The movement of the relay nodes leads to the problem of node programming that is described as Mobile Element Scheduling (MES). Frequent visiting of nodes will affect performance and there will be a minimum loss of data due to overflow of node storage. When a mobile relay node finds a node, it receives and stores the data in its storage by flushing the memory space of sensor for the next task.

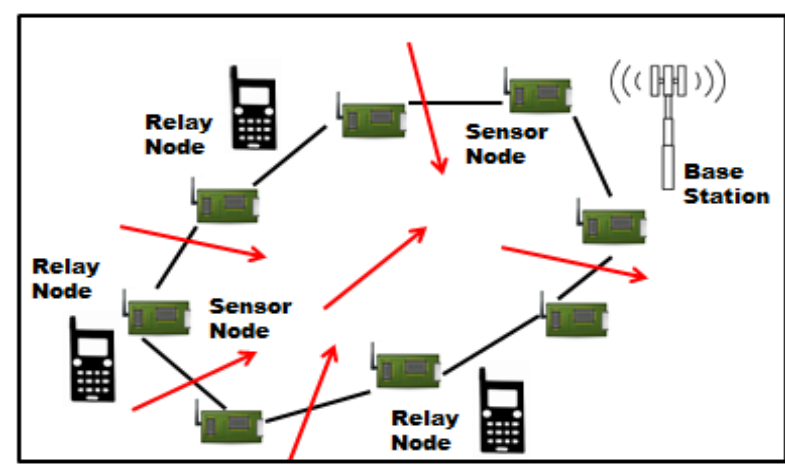

Fig. 1 Architecture with MRN and attacks can occur with barrier coverage problem

\subsection{Configurations of the Mobile Relay Node}

The main elements of the MRN in WSN are:

- The nodes present are the main source of data and reach the survey as their major task. In addition, they pass data to the network, according to the communication paradigm adopted.

- Mobile relay nodes execute a task specified, acting as intermediary data gateway. They stay not as the source or target of a message, but use mobility to maintain the lifetime of network.

- The multiple sinks (or base station) are the destination to store aggregated data from nodes incidentally (i.e. by intermediary nodes) or by direct (i.e. visit by sensors and data collection from all of them) and sent in parallel or concerned users by an Internet connection.

\section{PROBLEM STATEMENT}

In this paper, the sensor network contains nodes of uniform energy sensors. The sensor nodes are configured with the limited-range wireless interface. Distributed nodes detect events that occur in their environment and store them in the buffer.

\subsection{Assumptions}

The hypotheses related to the MRN and static nodes are discussed below.

- The typical size of the MRN and sensor nodes is insignificant. The problem of barrier coverage can be solved by implementing the node along the intended intrusion route.

- Each sensor has a similar size of temporary storage, with time $t=0$, every sensor node's memory starts with null state. The MRN contains adequately vast amount of memory for data, so there is no buffer overflow problem.

- The time to exchange data between the MRN and the sensor nodes is not insignificant, unlike latency due to the movement of the MRN. The data transmission rate is indicated by $D t r$.

- MRN moves on any route, excluding any delay during the turn. 


\subsubsection{Definitions}

Obstruction: A barrier / barrier area requires mechanisms to detect obstacles and strategies to avoid them, as well as ensuring the required coverage.

Uniform and regular implementation: Regular and uniform implementation [14] reduces the energy consumed during the data collection phase and minimizes the delay in data collection. It also provides better consistency of the time and space of the measurements notified to the sink.

Relation between the sensing range $r$ and the transmission range $R$.

This study impacts on the choice of the solution. If, for example, $R \geq \sqrt{3} r$, it is sufficient to resolve the coverage problem to get connectivity as a result of the coverage. If the transmission interval $\mathrm{R}$ is strictly lower than the detection range $\mathrm{r}$, a deployment would require a lower target distance between the sensor nodes than required by the total area coverage. Therefore, a vast number of sensor nodes used, leads to a more expensive solution.

\section{MOBILE RELAY NODE CONSTRAINT FOR DATA TRANSMISSION}

In this approach, the problem of barrier coverage can arise when the distribution scenario considers blocking of the phenomenon as a physical tetrahedron object, such as the terrain or the huge rock that could be encountered during data transmission between the sensor nodes. Let's understand how this object will have an impact on the efficiency between the groups of nodes.

Theorem 1: It is said that a tetrahedron is an isosceles, if and only if, the sum of the angles of the face at each vertex of the polyhedron is $180^{\circ}$.

Let a tetrahedron have the length of one side and let its base lie in the plane $z=0$ with a vertex placed along the positive $\mathrm{x}$ axis.

The vertices for the polyhedron of this tetrahedron are in $(x, 0$, $0),(-d, \pm a / 2,0)$, and $(0,0, h)$, where the following equation represents the conditions embedded in an object.

$\mathrm{x}=\frac{\mathrm{a} / 2}{\cos \pi / 6}=\frac{1}{3} \sqrt{3} \mathrm{a}$

$d=\sqrt{x^{2}-\left(\frac{1}{2} a\right)^{2}}=\frac{1}{6} \sqrt{3} a$

$h=\sqrt{\left(a^{2}-x^{2}\right)}=\frac{1}{3} \sqrt{6} a$

$$
A=\frac{1}{2} a(R+x)=\frac{1}{4} \sqrt{3} a^{2}
$$

An isosceles tetrahedron is an irregular tetrahedron where each pair of opposing edges of the polyhedron is equal, i.e. $p^{\prime}$ $=p, q^{\prime}=q$ and $r^{\prime}=r$, so that all triangular faces are congruent. It is said that a tetrahedron is isosceles if and only if the sum of the angles of the face at each vertex of the polyhedron is $180^{\circ}$ and if and only if it's inner sphere and circumsphere are concentric.

The volume of an isosceles triangle is given by

$\mathrm{V}=\sqrt{\frac{\left(p^{2}+q^{2}-r^{2}\right)\left(p^{2}+r^{2}-q^{2}\right)\left(q^{2}+r^{2}-p^{2}\right)}{72}}$

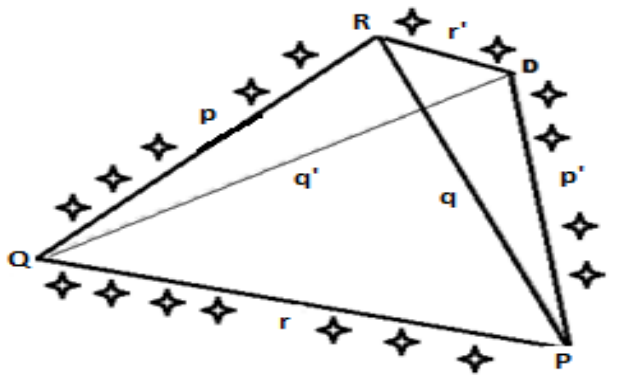

Fig. 2 Tetrahedron physical object as terrain or rock

The height of the tetrahedron is between the center of the basic triangle and the vertex shown below.

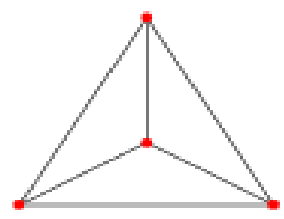

$$
h=\operatorname{sqrt}(3) / 2 * a
$$

(6)

This height ' $h$ ' is calculated using Pythagoras theorem. Thus circum-radius $\mathrm{R}$ is found from the equation below.

$$
R=\frac{x^{2}+h^{2}}{2 h}=\frac{1}{4} \sqrt{6} a=0.6123 a
$$

The global coverage for a sensor network is the combination of numerous low cost sensor coverage areas so that the coverage is more flexible and can be properly adjusted by adding the new nodes or mobile relay nodes as shown in Fig.2, wireless sensor networks can also cover hostile lands (such as battlefields, swamps, etc.). in case of non-availability of infrastructure and/or implementation is not feasible.

\section{SPLIT AND SCHEDULE (SS) ALGORITHM}

Suppose $C j, j=1 \ldots P$ denotes group $\mathrm{j}$, where $\mathrm{P}$ is the sum of all groups. In the SS algorithm, the sensor nodes are initially subdivided into clusters so that the number of times overflow of the nodes for $j>i>0$ and cluster $C i$ is less than that of $C j$. In addition, the node interval will always be twice the number of times overflow of nodes in $\mathrm{Ci}+1$. Thus, nodes in there often visit the number of times that nodes in $\mathrm{Ci}+1$ take to reach the nodes in creation. Each cluster is further subdivided into sub-cluster so that nodes are geographically closer to the adjacent cluster in the same sub-cluster. This type of two-level cluster deployment leads to a set of nodes that have the same time and position limit. Therefore, in each subgroup, the time needed to reach the nodes can also be used as a remedy to the travelling salesman problematic (TSP). Finally, the below steps for specific clusters is combined to build a complete list that ensures compliance with all restrictions.

- Step 1: Split the nodes ' $\mathrm{n}$ ' into clusters of $\mathrm{P}$ in relative to buffer storage overflow times.

- $\quad$ Step 2: Depending on the position of each cluster $\mathrm{Cj}$ into 2(j-1) sub-clusters with Split \& Schedule algorithm.

- $\quad$ Step 3: The minimum weighted path for each sub cluster.

- $\quad$ Step 4: Concatenate all visited route with minimum weights to make the complete schedule. 


\subsection{Sensor nodes deployed using two Dimensional KD- trees}

Consider an example of two-dimension KD tree presented in fig. 2 earlier for sensor nodes distributed randomly in a specific location. As depicted above in the fig. 3, the group of sensor nodes is equally divided into two components without distortion along its $\mathrm{x}$ axis. Nodes along with the $\mathrm{x}$-axis are smaller than the average axis-of-x assigned to one part and the rest to the other KD tree.

The KD [20] tree algorithm depicted above is used to run this partition. It is a k-dimension tree for retrieving data through self-coordinated exploration. In the situation here, $2 \mathrm{D}$ tree is considered in which distance and breadth are the two dimensions of the deployed sensor network. Since the split portion performed vertically and horizontally is in two slices taking $y$-axis of the deployed nodes. The same procedure is continued alternately unless the specified amount of divisions is obtained. The amount of split correspondingly indicates the sum of subclasses in the taken problem and determines the complexity of the two-dimensional tree.

Now the SS algorithm proposes that, the cluster $\mathrm{Cj}$ has been divided into two subgroups of ( $\mathrm{j}-1)$, so the complexity of the $2 \mathrm{D}$ tree for $\mathrm{Cj}$ has been defined as:

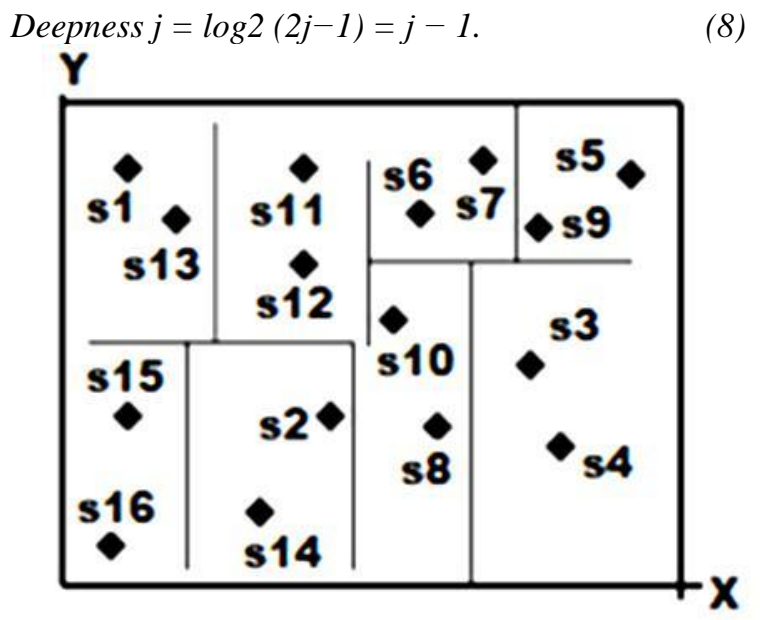

Fig. 3 Sensor Nodes deployed sliced in two Dimensional KD- tree

2D-tree (L, slice-x, Deepness, D)

1 : if $\mathrm{D}=$ Deepness then

2: revert

3: end if

4: if slice-along $x$ then

5: arrange $\mathrm{L}$ conferring to $\mathrm{x}$ axis

6: else

7: arrange $\mathrm{L}$ conferring to $\mathrm{y}$ axis

8: end if

9: split L into L1 and L2

10: slice-along $\mathrm{x} \longleftarrow \sim$ slice-along $\mathrm{x}$

11: KD-tree (L1, slice-along $\mathrm{x}$, deepness, $\mathrm{D}+1)$

12: KD-tree (L2, slice-along $\mathrm{x}$, deepness, $\mathrm{D}+1$ )

The input $\mathrm{L}$ is the list of nodes in the cluster to be split. Slice$\mathrm{x}$ is a flag (boolean) showing the condition for slice: $\mathrm{x}$ position (true) or y-position (false). ' $\mathrm{D}$ ' is the current sliced level and deepness is the depth of the KD-tree that is estimated by specific number of sub-clusters. In each call for process, initially the nodes are arranged with respect to $\mathrm{x}$ or $\mathrm{y}$ axes and splitted into two relative to the value of slice-x. Then, slice-x flag is altered and the process is invoked recursively on resulting split made until the chosen 2D-tree deepness is reached.

\section{PERFORMANCE EVALUATION}

The performance of the split and scheduling algorithm (SS) along with existing MES and MWVS algorithms are implemented using simulations under different scenarios considering various test cases. The experimental configuration is implemented using NS2, the sensor nodes being distributed evenly in a boundary of 1000 sqm. The mobility of nodes is executed with $2 \mathrm{~ms}$ and the weights are similar as that of hops between the nodes visited.

\subsection{Performance Measuring Terms}

In order to evaluate the performance of the proposed algorithm the following metrics are used:

- Coverage Percentage [17]: The coverage proportion is measured as the ratio of area covered to the total area of the terrain. Coverage has been one of the important limitations in directional sensor network and thus a higher percentage ratio of coverage is expected.

- Network Lifetime [18]: The network lifetime is defined as the difference from the time at which the first node dies out of energy to the deployed time of the nodes in network.

- End-to-end delay: The delay comprises of the transmission, waiting and touring type of delays for the transmitting a designated packet through a network from source to sink [19].

- Data Loss is described as the ratio of the data lost relative of overflow of buffer to overall volume of generated data.

- Transmission overhead termed as the efficiency and overall cost for the algorithm; less transmission indicate less congestion and more energy conserve, as most of mobile/sensor nodes are battery-operated.

\subsection{Simulation Results}

This sector discusses the overall performance of the algorithm proposed with respect to metrics to solve the problem of barrier coverage with existing methods. The fig. 4 depicts the variation of node density in relation to network lifetime. Here the nodes are deployed with uniform scalability of range of 10 nodes. Each time the nodes are added to network it affects the energy consumption and gradually falling above the threshold of $40 \%$ margin. Thus, SS algorithm achieves better longevity than the other two algorithms during scalability of nodes.

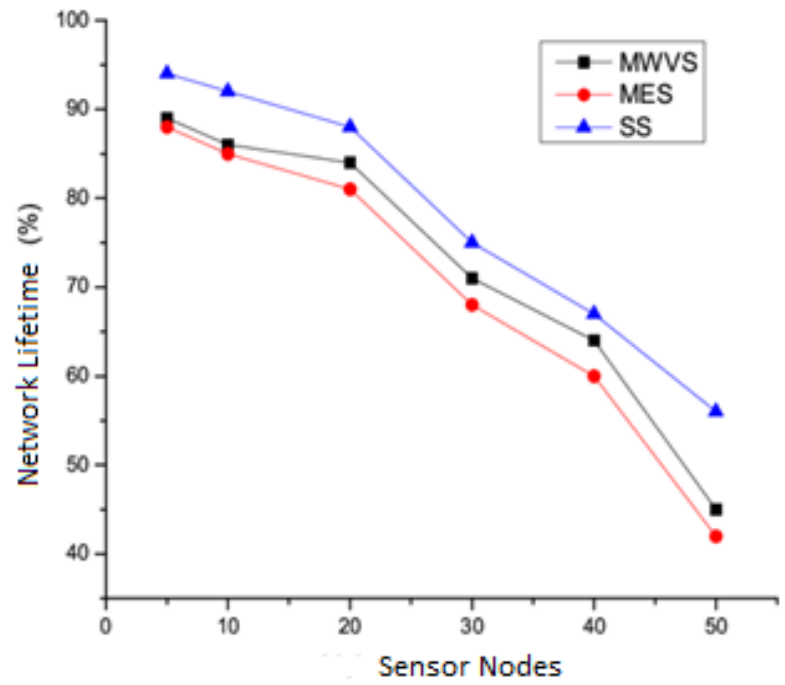

Fig. 4 Node Density and its Lifetime 
The fig.5 shows the packet transmission error 'Perr' that began in deployment phase in presence of an object. The cluster formed initially before transmission has reduced the 'Perr' to certain extent towards the variation of nodes deployed.

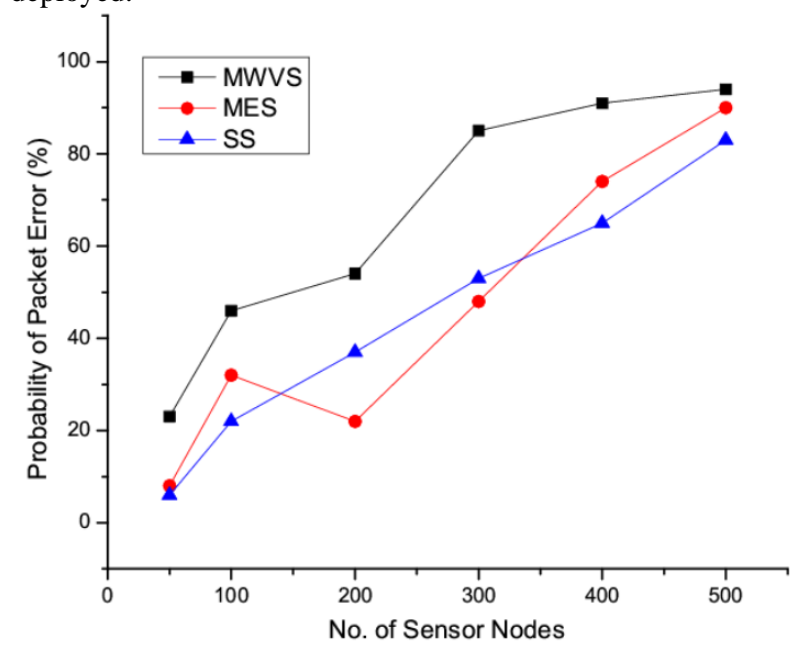

Fig. 5 Node Density with Transmission Error

There is an overlap at one stage in error rate between two algorithms due to mobility and sudden increase in node density. At initial stage the graph of SS algorithm shows a linear variation and outplays with certain less Perr against the either of the existing algorithms MES \& MWVS. In fig. 6, it displays the comparison of proposed SS algorithm in different scenarios. There are two deployment scenarios considered, one using an obstruction and other without an obstruction such as rock or terrain. In both the scenario, there are set of increasing and decreasing coordinates, since the energy is consumed for cluster formation and initial setup for the exchange of data. The total number of packets delivered effectively can be observed that on an average rate as $70 \%$ when compared with the related work discussed which is much lesser around $50 \%$.

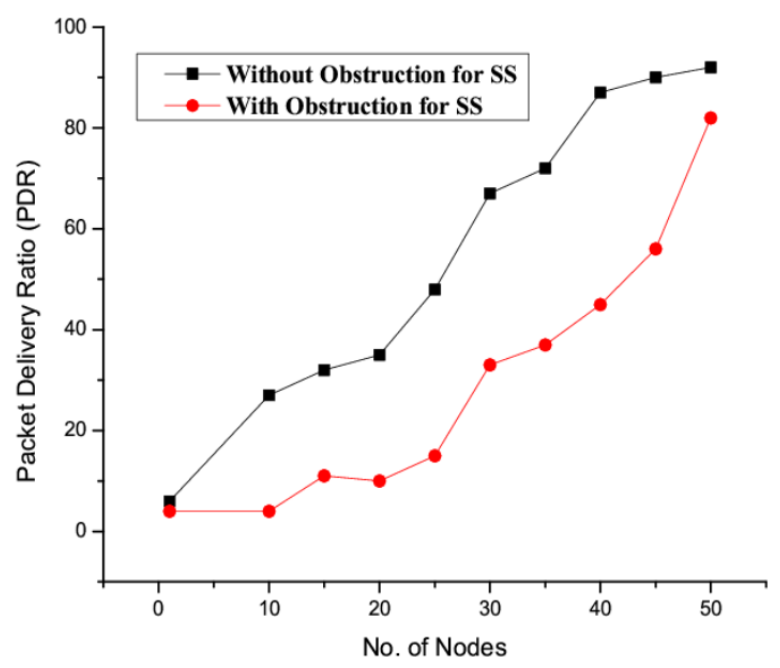

Fig. 6 Number of nodes and Successful Transmission

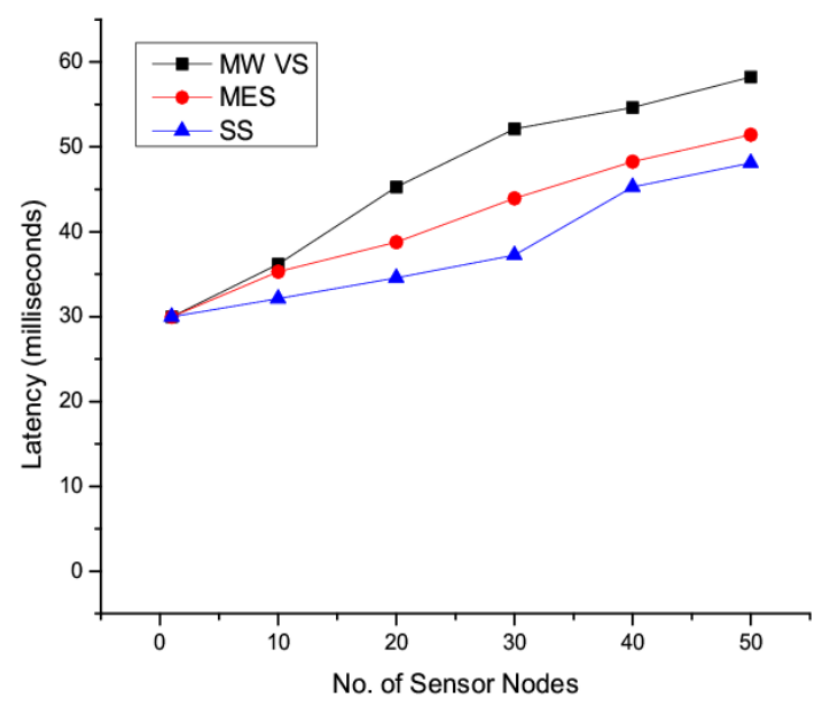

Fig. 7 Node Density and its End -End Delay

Further the performance is analyzed using the latency factor against the number of nodes with multiple sink. The fig.7 presents the three algorithms SS, MES and MWVS in visiting the nodes and making faster and secure transmission among which the SS algorithm takes less amount of time to complete the session with maximum of $40 \mathrm{~ms}$. The latter approaches consume more resources and time in achieving the task assigned.

\section{CONCLUSION}

Most of previous papers disregarded the energy consumption by mobile relay nodes (MRN). Hence, a holistic approach is proposed to reduce the overall energy consumed by relay nodes movement and formation of barrier of nodes. An iterative tactic is developed to compute the finest positions of relay nodes that are implemented in a distributed fashion. The proposed SS algorithm prevents attacks that arise in an unfriendly environment effectively. The deployment strategies and role of MRN considered has led to the growth in lifetime of network and solves the energy crisis which is a major challenge in the coverage problem. This work can be further extended using different mobility models for other coverage problems.

\section{REFERENCES}

[1] I Akyildiz, W Su, Y Sankara Subramaniam and E Cayirci, 2002. "Wireless Sensor Networks: a Survey", IEEE Transactions on Computer Networks, no. 38, pp. 393-422.

[2] Fall K, 2003. "A Delay-Tolerant Network Architecture for Challenged Internets", In Proceedings of the 2003 Conference on Applications, Technologies, Architectures, and Protocols for Computer Communications (SIGCOMM 2003), pp.27-34.

[3] Shah, R. C., Roy, S., Jain, S., Brunette, W, 2003. "Data mules: Modeling A Three-Tier Architecture for Sparse Sensor Networks", In Proceedings of the 2nd ACM International Workshop on Wireless Sensor Networks and Applications (SNPA 2003) pp. 30-41.

[4] Chakrabarti A, Sabharwal A, Aazhang B, 2005, "Using Predictable Observer Mobility for Power Efficient Design of Sensor Networks", in Proceedings of the 2nd International Workshop on Information Processing in Sensor Networks, pp. 129-145.

[5] Ekici, E., Gu, Y., Bozdag, D, 2006, "Mobility-Based 
Communication in Wireless Sensor Networks", IEEE Communications Magazine vol.44, no.7, pp. 56-62.

[6] Gandham, S., Dawande, M., Prakash, R., Venkatesan, S, 2003, "Energy Efficient Schemes for Wireless Sensor Networks with Multiple Mobile Base Stations", in Proceedings of 46th IEEE Global Telecommunications Conference Workshops, pp.377-381.

[7] Wang, Z. M., Basagni, S., Melachrinoudis, E., Petrioli.C, 2005, "Exploiting Sink Mobility for Maximizing Sensor Networks Lifetime", In Proceedings of the 38th Hawaii International Conference on System Sciences (HICSS).

[8] Srinidhi T, Sridhar G, Sridhar V, 2006, "Topology Management in Ad Hoc Mobile Wireless Networks", in Proceedings of the 24th IEEE International Real-Time Systems Symposium, pp. 29-32.

[9] Wu, J. Yang, S, 2005, "Smart: A Scan-Based Movement Assisted Sensor Deployment Method in Wireless Sensor Networks", In Proceedings of the 24th IEEE Conference on Computer Communications (INFOCOM), vol. 4, pp.2313-2324.

[10] Wang, G., Cao, G., La Porta, T., Zhang, W, 2005, "Sensor Relocation in Mobile Sensor Networks", in Proceedings of the 24th IEEE Conference on Computer Communications, vol. 4, pp.2302-2312.

[11] Tang, C., McKinley P, 2006, "Energy Optimization Under Informed Mobility", IEEE Transactions on Parallel and Distributed Systems vol.17, no.9, pp.947962.

[12] [12] El-Moukaddem, F., Torng, E., Xing, G., Kulkarni, S. 2009, "Mobile Relay Configuration in Data-Intensive Wireless Sensor Networks", in Proceedings of the 6th IEEE International Conference on Mobile Ad Hoc and Sensor Systems, pp.80-89.

[13] [13] Dini, G., Pelagatti, M., Savino, I. M, 2008, “An Algorithm for Reconnecting Wireless Sensor Network Partitions", in Proceedings of the 5th European conference on Wireless Sensor Networks (EWSN), pp.253-267.

[14] [14] A. A. Somasundara, A. Ramamoorthy, M. B. Srivastava, 2004, "Mobile Element Scheduling for Efficient Data Collection in Wireless Sensor Networks with Dynamic Deadlines", in 25th IEEE International Real-Time Systems Symposium, pp.296-305.

[15] [15] G. Xing, J. Wang, K.Shen, Q.Huang, X.Jia H.C.So, 2008, "Mobility Assisted Spatial-Temporal Detection in Wireless Sensor Networks", Proc. 28th International Conference on Distributed Computing Systems (ICDCS), Beijing, China, June 17-20.

[16] [16] D. Wang, J. Liu, Q. Zhang, 2007, "MobilityAssisted Sensor Networking for Field Coverage", In
Proceeding of IEEE Global Communications Conference, pp.1190-1194.

[17] [17] Yaoyao Gu and D. Bozdag and E. Ekici and F. Ozguner, 2005, Chang-Gun Lee, "Partitioning Based Mobile Element Scheduling in Wireless Sensor Networks", in Second Annual IEEE Communications Society Conference on IEEE SECON, pp. 386-395.

[18] Sharmin, Selina, Fernaz Narin Nur, Md. Abdur Razzaque, Md. Mustafizur Rahman, 2015, "Network lifetime aware area coverage for clustered directional sensor networks", International Conference on Networking Systems and Security (NSysS).

[19] Zhao, W. Ammar, M. "Message Ferrying: Proactive Routing in Highly-Partitioned Wireless Ad Hoc Networks", 2003, In Proceedings of the 9th IEEE International Workshop on Future Trends of Distributed Computing Systems, pp.308-314.

[20] J. L. Bentley, 1985, "Multidimensional Binary Search Trees used for Associative Searching," Communications of the ACM, vol. 18, no. 9, pp. 509-517.

\section{AUTHOR'S PROFILE}

$\mathrm{C} \mathrm{N}$ Abhilash is currently working as an Associate Professor in the Department of ISE, SJB Institute of Technology, Bengaluru, India. He has 11 years of teaching experience. Currently he is pursuing Ph.D in the Department of CSE, UVCE, Bangalore University, Bengaluru. His research area interests are Computer Networks, WSN and Ad hoc Networks.

S H Manjula is currently Associate Professor, Department of Computer Science and Engineering, UVCE, Bangalore University, Bengaluru. She obtained her B.E., M.E. and Ph.D in CSE. She published book on Wireless sensor Networks. She published more than 30 papers in refereed International Journal and Conference papers. Her research interests are in the field of Wireless Sensor Networks, Semantic web and Data mining.

$\mathrm{K}$ R Venugopal is currently the Special Officer, DVG Jnanavahini University and Principal, UVCE, Bangalore University, Bengaluru. He was awarded Ph.D. in Economics from Bangalore University and Ph.D. in Computer Science from IIT, Madras. He has a distinguished academic career and has degrees in Electronics, Economics, Law, Business Finance, Public Relations, Communications, Industrial Relations, Computer Science and Journalism.

He has authored/coauthored/edited 29 books. His books are published by Springer Verlag, Mc. Graw Hill etc. He has over $250+$ research papers to his credit. His research interests include Computer Networks, Wireless Sensor Networks, Parallel and Distributed Systems, Digital Signal Processing and Data Mining. 